Referencia para citar este artículo: Valbuena-Espinosa, J. C. (2018). Criterios para el análisis y evaluación de casos en la educación moral. Revista Latinoamericana de Ciencias Sociales, Niñez y Juventud, 16(2), 685-694. doi:https://doi.org/10.11600/1692715x.16203

\title{
Criterios para el análisis y evaluación de casos en la educación moral*
}

\author{
JUAN CAMILO VALBUENa-ESPINOSA** \\ Profesor Fundación Universitaria Juan de Castellanos, Colombia.
}

\begin{abstract}
Artículo recibido en julio 7 de 2017; artículo aceptado en octubre 17 de 2017 (Eds.)
\end{abstract}
\begin{abstract}
- Resumen (descriptivo): el objetivo central del artículo es proponer unos criterios para el análisis y evaluación de casos en la educación moral. Los criterios que se proponen son cuatro: 1) la identificación de los dilemas morales que se encuentran en la narración del caso; 2) el análisis de las circunstancias y hechos; 3) la consistencia argumentativa; y 4) la justificación moral de las alternativas de decisión. Estos criterios sirven para orientar los procesos pedagógicos en el aula a partir de casos con dilemas que fortalecen el desarrollo del juicio moral. Los docentes pueden adoptar los criterios para orientar sus clases y evaluar el desempeño de sus estudiantes. Los criterios propuestos pueden ser implementados tanto en la educación básica y media como superior.
\end{abstract}

Palabras clave: ética, método de enseñanza, estudio de caso, educación moral, desarrollo moral, dilemas morales (Tesauro de la Unesco).

\section{Criteria for the analysis and evaluation of cases in moral education}

- Abstract (descriptive): The central objective of the article is to propose different criteria for the analysis and evaluation of cases in moral education. The article proposes four criteria: 1) the identification of moral dilemmas that are identified in the narration of the case; 2) the analysis of facts and circumstances; 3) argumentative consistency and 4) the moral justification of the alternatives for moral decisions that students identify. These criteria serve to guide pedagogical processes in the classroom through cases with dilemmas that strengthen the development of students'moral judgment. Teachers can adopt these criteria for use in their classrooms and to evaluate the performance of their students. The proposed criteria can be implemented in both secondary and higher education.

Key words: Ethics, method of teaching, case studies, moral education, moral development, moral dilemmas (Thesaurus Unesco).

\section{Critérios para a análise e avaliação de casos na educação moral}

- Resumo (descritivo): o principal objetivo do trabalho é propor critérios para a análise e avaliação de casos na educação moral. Os critérios propostos são quatro: 1) a identificação de dilemas morais encontrados na narrativa do processo; 2) Análise das circunstâncias e fatos; 3) consistência argumentativa e 4) a justificação moral das alternativas decisão moral feitas pela

\footnotetext{
Este artículo corto hace parte del proyecto denominado «Formación ética en la educación básica y media: Estrategias y acciones» inscrito al Banco de Proyectos de la Dirección General de Investigación e Innovación de la Fundación Universitaria Juan de Castellanos con el código C10521. El proyecto se encuentra en realización desde el año 2016 y tiene fecha de terminación en el año 2018. Gran área de conocimiento: Ciencias Sociales; área de conocimiento: Ciencias de la Educación.

** Profesional y Magíster en Filosofía de la Universidad del Rosario. Orcid: 0000-0001-8467-8107. Correo electrónico: jcvalbuena@jdc.edu.co
} 
mesma. Estes critérios servem para orientar os processos de aprendizagem na sala de aula a partir de casos com dilemas que fortalecem o desenvolvimento do juizo moral. Os professores podem adotar os critérios para orientar suas aulas e avaliar o desempenho de seus alunos. Os critérios propostos podem ser implementados tanto no ensino primário e secundário como superior.

Palavras-chave: ética, método de ensino, estudo de caso, educação moral, desenvolvimento moral, dilemas morais (Thesaurus da Unesco).

-Introducción. -Identificación de los dilemas morales. -Análisis de las circunstancias y hechos. -Consistencia argumentativa. -Justificación moral de las alternativas de decisión. -Criterios de evaluación para análisis de caso. -Conclusiones. -Lista de referencias.

\section{Introducción}

Una de las herramientas pedagógicas que actualmente se utiliza con frecuencia por parte de docentes de diversas áreas y niveles educativos es el uso del método basado en casos. Los casos se pueden entender como narrativas que incluyen información y datos psicológicos, sociológicos, científicos, antropológicos, históricos y de observación (Wassermann, 2006). A partir de esas narrativas se espera promover en los estudiantes mayor sentido crítico frente a los temas y problemas éticos; fortalecer el análisis de situaciones dilemáticas para provocar cambios positivos en la capacidad para tomar decisiones acertadas; y aumentar la motivación del estudiante mediante el intercambio de opiniones, actitudes y creencias con sus compañeros en un proceso de debate (Ewing, 1990).

En el contexto de la educación moral, los casos son instrumentos útiles porque ayudan a que tanto el docente como los estudiantes tengan un mayor acercamiento a los debates actuales de la ética y puedan realizar análisis más agudos; así mismo, inducen al esfuerzo por obtener una mayor compresión del universo de lo ético y permiten el desarrollo del pensamiento crítico (Estrada \& Alfaro, 2015).

Según Puig y Martín (2015), los casos harían parte del conjunto de prácticas morales que constituyen lo que ellos denominan un currículo de educación en valores. En términos generales, las prácticas morales son aquel conjunto de actividades educativas que «ayudan a adquirir la competencia para valorar la actualidad y para discriminar lo que se debe aceptar y cómo adquirirlo, lo que se debe transformar y como cambiarlo, y lo que se debe inventar y como crearlo» (p. 11). Dichas prácticas, a su vez, se pueden distinguir en su intencionalidad en procedimentales o sustantivas. Los casos con una estructura dilemática harían parte de las prácticas procedimentales, ya que estimulan el proceso de deliberación en torno a problemáticas morales, permitiendo la diversidad de opiniones ante una situación en la que se presenta una confrontación de valores, alentando la posibilidad establecer disensos y análisis grupales sobre los pros y contras de los cursos de acción, la valoración de consecuencias y, sobre todo, estimulando la búsqueda de soluciones argumentadas (Puig \& Martín, 2015).

En ese sentido, el presente texto busca presentar una serie de criterios generales que pueden ser acogidos por los docentes para orientar a los estudiantes a la hora de abordar el análisis de un caso de naturaleza ética. Y esos mismos criterios también pueden servir para evaluar la manera cómo los estudiantes se apropian del caso y lo analizan. Los criterios propuestos fueron seleccionados no solo a partir de una revisión documental (Hirsch, 2006), sino a partir de los aportes de docentes que orientan asignaturas de ética y valores tanto en la educación básica y media como en la educación superior, en las ciudades de Tunja y Bogotá. Esta información se ha venido recolectado y recopilando desde año 2016. Los aportes de los docentes han sido extraídos de grupos focales y ejercicios piloto donde los maestros han manifestado sus ideas acerca de cómo estructurar el análisis de casos en el contexto de sus clases y cómo evaluar el desarrollo de dicho análisis por parte de los estudiantes.

Desde una concepción de la educación moral basada en el desarrollo del juicio moral y la apropiación de valores, los criterios que se proponen en el artículo parten de la aspiración que tanto 
en la enseñanza como el aprendizaje moral deben estar orientados a adoptar y promover prácticas de deliberación. Entendiendo que la deliberación significa:

reflexionar conjuntamente sobre una cuestión, es decir, establecer los hechos, analizar las causas que los provocan, comprender las posiciones de los implicados, analizar los motivos y las intenciones de todos ellos, valorar las consecuencias, dialogar aportando las mejores razones que pueda aducir cada uno de los puntos de vista en litigio, buscar soluciones mejores, reconocer las dificultades para encontrar puntos de acuerdo, pero reconocer también la amplitud de perspectivas que se han podido obtener durante el proceso. (Puig \& Martín, 2015, p. 13)

Como vemos, el ejercicio de deliberación exige una seria de competencias argumentativas, comunicativas y sociales para abordar moralmente situaciones problemáticas de forma adecuada. En ese sentido, los criterios que se seleccionaron para orientar el análisis y la evaluación de casos en la enseñanza de la ética se soportan en la idea que la deliberación sea reflexiva en tanto lleve a la formulación de un juicio en donde se evalúa una situación, o bien conduzca a la descripción de un curso de acción que se considere correcto en relación con una situación determinada. La formulación de juicios morales, como señalaba Kohlberg (1984) y Rest (1979), implica el ejercicio de varias competencias. La primera de ellas es el ejercicio de la autonomía, en el sentido que la persona sea capaz de justificar argumentativamente ante sí mismo y ante los otros sus opiniones morales y decisiones. Al respecto, Puig y Martín (2007) señalan que, ante situaciones moralmente problemáticas, los agentes morales reflexivos «aportan razones para justificar la corrección e incorrección de opiniones y conductas relacionadas con este tipo de situaciones» (p. 157). La segunda competencia se relaciona con el análisis cuidadoso de las circunstancias en las cuales se encuentra la persona que tiene que decidir. En otras palabras, un juicio moral no debe ser ingenuo, sino que debe ser informado en la medida que facilita una comprensión crítica del contexto relacionado con el caso. Este tipo de comprensión exige mirar en cada caso los condicionamientos políticos, legales, culturales, sociales e incluso naturales que determinan la viabilidad de las alternativas de acción disponibles. La tercera competencia involucra la capacidad de argumentar en situaciones moralmente problemáticas y, por eso, abarca las dos competencias anteriores, ello porque la capacidad para argumentar no se desarrolla de manera monológica en un diálogo consigo mismo, sino que se requiere examinar seriamente las opiniones de los otros, para lo cual es necesario el intercambio de ideas y razonamientos. La deliberación moral no es un procedimiento dogmático; requiere poner en cuestión las propias creencias, y, por ello mismo, no considera a priori que las posiciones de los otros por ser diferentes a las propias opiniones sean incorrectas. Desde Platón y Aristóteles se ha considerado que la deliberación moral es un esfuerzo cooperativo, que como tal se realiza a través del diálogo con los otros, del contraste crítico entre las distintas posturas alrededor de un problema específico.

Cabe señalar que los criterios propuestos y su aplicabilidad se consideran pertinentes para estudiantes de últimos grados de educación escolar o superior, donde se espera que los educandos por edad y nivel académico tengan una serie de competencias argumentativas y unos saberes que les ayuden a relacionarse y a comprender mejor los casos dilemáticos propuestos por los docentes. Para promover el proceso de deliberación y justificación moral a la hora de abordar el análisis de casos es importante tener en cuenta los posibles cursos de acción, principios y justificaciones que deben ser tenidos en cuenta y aplicados. En ese ejercicio el centro del análisis se orienta indagar sobre cuál sería la mejor justificación y línea de acción a seguir.

Para abordar el análisis del caso y la evaluación que hacen los estudiantes se pueden identificar por lo menos cuatro criterios: 1) la identificación de los dilemas morales que se encuentran en la narración del caso; 2) el análisis de las circunstancias y hechos; 3) la consistencia argumentativa; y 4) la justificación moral de las alternativas de decisión. Estos criterios serán explicados con más detalle a continuación. 


\section{Identificación de los dilemas morales}

En la discusión sobre las orientaciones teóricas de la educación moral hay posturas que, siguiendo los estudios de Kohlberg (1984), consideran que el aprendizaje ético no puede reducirse a una serie de teorías o preceptos generales de conducta, sino que un aspecto importante de ese aprendizaje es el mejorar el juicio moral y ayudar al proceso de deliberación para la toma de decisiones ante un dilema moral (Carrillo, 1982; Hernández, González, \& Pérez, 2015; Martínez, Navarro, \& Pérez, 2015; Meza, 2008; Noam, 1985).

Un juicio moral necesita para su adecuado análisis de una descripción de la situación y el contexto frente a la cual se tiene que tomar una decisión. Para realizar el ejercicio de análisis es necesario identificar los intereses que están en conflicto y las circunstancias relevantes que llevan a ese conflicto de intereses. Cabe recordar que un dilema ético puede tener la siguiente estructura: «a) parte de la evidencia indica que el acto $x$ es moralmente correcto, y otra que es moralmente incorrecto, pero ambos casos la evidencia no resulta concluyente (...) y b) un agente considera que moralmente debería y no debería realizar el acto $x \gg$ (Lemmon, 1962, pp. 139-158).

Adicionalmente a la anterior descripción, cabe señalar que un dilema también se caracteriza porque en cada una de las alternativas disponibles los agentes involucrados tienen razones morales de peso tanto para seguirlas como para dejarlas de lado. En este tipo de situaciones cualquiera que sea la alternativa que el agente escoja podrá juzgarse como justa o injusta, correcta o incorrecta, de acuerdo a la razón moral que se esté utilizando para justificarla. En ese sentido hay que tener presente que un dilema moral puede involucrar tres perspectivas de reflexión (Bermúdez \& Jaramillo, 2000): la pragmática, la teleológica y la de la justicia. La perspectiva pragmática se orienta por una concepción teleológica de la ética, donde se evalúa la relación entre los medios y los fines en las acciones humanas. La pregunta central que orienta el debate en esa perspectiva es: ¿qué debo hacer para alcanzar $x$ ? En ese caso se reflexiona sobre los medios adecuados para alcanzar ciertos fines. La segunda perspectiva aborda la pregunta: ¿cuál es la mejor forma de vivir?, y para ello se aborda la reflexión acerca de la felicidad y la valoración que el sujeto le da a su proyecto de vida para alcanzar dicho fin. La tercera perspectiva aborda la cuestión de las éticas deontológicas que se centran en la justificación moral del sentido de lo justo y del deber. En dicha perspectiva ya se involucran temas institucionales, políticos y legales relacionados con derechos, normas, principios y prácticas. En la perspectiva de la justicia también pueden estar involucradas las dos perspectivas anteriores. Por ejemplo, en un caso sobre la justicia pueden surgir cuestionamientos sobre si es aceptable que una persona actué ilegalmente para resolver problemas económicos. Dicho cuestionamiento lleva al debate de por qué ciertos fines son deseables y qué medios son legítimos para alcanzarlos (primera perspectiva). También involucra la discusión sobre la concepción de felicidad (segunda perspectiva) y por qué para algunas personas la felicidad se asocia con la riqueza material. Finalmente, la perspectiva de la justicia llevaría a los participantes del dilema a preguntar si es justo actuar como bandido para satisfacer necesidades materiales particulares, incluso en aquellos casos en los que esas necesidades parecen apremiantes.

\section{Análisis de las circunstancias y hechos}

Una vez identificada la estructura del dilema, se debe proceder a tener en cuenta las circunstancias, hechos y creencias que conforman el contexto del caso para proponer una posible forma de abordarlo. Esto exige una reflexión sobre los hechos más relevantes que hacen parte del dilema y la forma en que estos inciden o afectan a los agentes. Para lograr una adecuada reflexión del dilema se sugiere que los estudiantes tengan la posibilidad de documentarse acerca de los referentes legales, históricos, culturales o sociológicos que están relacionados con las circunstancias o hechos que hacen parte del caso. Así la reflexión del dilema no queda como un ejercicio artificial, sino que adquiere veracidad al identificar que es una situación que sucedió o que puede suceder en determinados contextos sociales 
(Ortega \& Mínguez, 1992). Para evitar que el ejercicio con dilemas morales parezca un ejercicio artificioso que no se relaciona con la experiencia de los estudiantes y que, por ende, no tiene mayor relevancia para ellos, se ha sugerido que los dilemas a trabajar en clase estén basados en hechos reales y no hipotéticos, que su narración se adapte a las necesidades curriculares y que no quede en un ejercicio académico aislado de una clase en particular (Linde, 2009).

También es importante en este análisis del contexto del caso que los estudiantes sean capaces de distinguir entre opiniones y hechos. Eso significa que el docente oriente a sus estudiantes para que sean cautelosos de no suponer aspectos que no sea evidentes, demostrables o sustentables a la hora de abordar los hechos o circunstancias. Por eso, el docente debe insistir en la adecuada contextualización del caso para que el ejercicio de análisis no quede en la mera opinión viciada por prejuicios, sino que esté respaldada en lo posible por evidencias que apoyen el juicio moral.

Al respecto algunos autores como Toulmin, Rieke y Janik (1979) y Habermehl (1976) han señalado que el análisis del razonamiento moral exige que las evidencias que apoyan el juicio sean exactas, pertinentes y completas. Cuando se habla que las evidencias sean exactas se entiende que los hechos del caso estén respaldados por datos confiables o pruebas científicas reconocidas. De esa manera, el debate que se desarrolle a partir del caso tendrá un sustento confiable y no caerá en el riesgo de convertirse en un intercambio de prejuicios que se basan informaciones tergiversadas, datos manipulados o pseudoteorías.

El criterio de la pertinencia hace referencia a que las evidencias no son datos o presupuestos aislados o generales que se pretenden aplicar a cualquier situación, sino que se consideran como pertinentes en la medida que tienen relación con los criterios morales y el contexto en el cual se desarrolla el dilema moral del caso. Por ejemplo, en una discusión sobre un dilema moral relacionado con el tema de la prostitución femenina en Colombia es posible que los datos que podamos exponer de la situación de la prostitución en países como Dinamarca o Suecia no sean relevantes para abordar el caso local, pues el contexto social y cultural colombiano es muy diferente al de dichos países; por lo tanto, los datos de esos contextos no brindarán las herramientas para hacer comparaciones o proponer alternativas de análisis.

Adicionalmente, las pruebas que sustentan el juicio moral deben ser completas en el sentido que se apoyan en toda la información que sean pertinente, de manera que no deben referirse de manera selectiva solo a pruebas que tienden a apoyar un único punto de vista (Velásquez, 2000). Esta condición es importante porque exige que los estudiantes asuman que en un dilema moral no hay una sola posición que cuente con más evidencias a favor, sino que pueden presentarse diversas posiciones con pruebas pertinentes que no deben desestimarse a la hora de analizar el caso.

\section{Consistencia argumentativa}

Una argumentación consistente para analizar un caso se identifica cuando las premisas del argumento son contundentes, es decir, son verdaderas y la conclusión es una consecuencia lógica de las premisas (Copi \& Cohen, 1990). Esta condición de la contundencia es una exigencia mínima porque un argumento que sustente una alternativa de decisión no puede partir de premisas falsas o de premisas que lleven a una conclusión que no se sigue. Otra condición mínima que hace parte de la consistencia argumentativa es el no cometer contradicciones lógicas entre los argumentos aducidos (Molina, 2010). Así, si alguien afirma por ejemplo todos los animales tienen derecho a la vida y después señala también que las vacas que son animales pueden ser sacrificadas para consumo humano, está incurriendo en contradicción lógica en la argumentación.

Una última condición que es necesaria para considerar que la argumentación del caso es consistente es que procure manejar un mínimo nivel de consistencia conceptual, en la cual se atribuyan a los mismos conceptos los mismos significados sin cambiarlos arbitrariamente en el desarrollo de la discusión (Molina, 2010). Un ejemplo de la consistencia conceptual es que, si discutimos acerca de 
la justicia en el caso de la objeción de conciencia de los médicos ante la práctica de un aborto legal, debería plantearse en el debate una aclaración de lo que significan conceptos relevantes como derecho o libertad. De tal manera que, en principio, los participantes del debate procuren usar un lenguaje común que facilite la discusión y ayude a llegar a unas conclusiones o diferendos, pero que sean producto de la argumentación y no de una confusión conceptual.

Los criterios descritos acerca de la consistencia argumentativa buscan que en el debate y análisis grupal de los dilemas los estudiantes eviten un uso retórico de los argumentos, en el sentido que el fin de la discusión sea persuadir a los destinarios mediante el uso de falacias (argumentaciones lógicamente incorrectas pero convincentes). Estas falacias generalmente apelan a criterios supuestamente válidos como la autoridad, la opinión de la mayoría o la descalificación del interlocutor. En contraste con el uso retórico de la argumentación, el análisis de casos debería promover la argumentación dialógica entendida como aquella que posibilita el diálogo entre perspectivas diferentes, donde se asume que una posición es válida si ofrece argumentos consistentes. Con ello se enseña que una postura y decisión ética ante un dilema es legítima en principio si cuenta con una argumentación suficientemente justificada (Erramouspe, 2004).

\section{Justificación moral de las alternativas de decisión}

La justificación moral se refiere a las razones que se pueden proponer con respecto a lo que se debería hacer frente a las alternativas que ofrece el dilema moral.

La evaluación y justificación del juicio moral no son la simple exposición de creencias y opiniones morales respecto a una situación en concreto, ya que puede suceder que estas no sean suficientes para argumentar que es mejor actuar de un modo y no de otro, o que no sean relevantes o aplicables al caso. El proceso de justificación moral se puede entender como el examen y la evaluación de los principios morales que sirven para sustentar las opiniones y las alternativas de decisión ante el dilema. En ese sentido, la justificación moral es un proceso cognitivo que permite la reflexión sobre los principios y valores, estableciendo una jerarquización que es fundamental para que la persona pueda resolver conflictos morales, no solo en el contexto de una asignatura de ética, sino en su vida cotidiana (Anaya \& Ocampo, 2016).

En el contexto de este artículo se asumirá que un principio moral es una guía general que orienta la formulación de juicios particulares en casos específicos y que ayuda explícitamente en el desarrollo de reglas y líneas de acción más detalladas (Beuchamp \& Childress, 2002).

En el proceso de examen de un principio moral es importante tener en cuenta que el principio se corresponda con las circunstancias descritas en el caso y que en la evaluación se oriente a establecer la mejor alternativa de acción a partir del principio moral escogido.

Para adelantar el proceso de examen del principio moral se debe revisar la consistencia entre el principio y el dilema. Por ejemplo, en el análisis de un dilema moral una persona considera (1) que es incorrecto desobedecer las órdenes de sus superiores que están justificadas en deberes contractuales y también esa persona cree (2) que es incorrecto colaborar con alguien que pone en riesgo la vida de otras personas inocentes. Luego el caso describe una situación donde se debe escoger entre obedecer al superior y evitar el incumplimiento de las obligaciones contractuales o desobedecerlo pero evitando poner en peligro la vida de un inocente. En esta situación el análisis del caso debe orientar a que la persona reconozca que ambos principios son incompatibles (no se pueden aplicar al mismo tiempo y situación) y, por ende, debe ponderarse cuál principio es más importante, ya sea por las consecuencias que derivan de su seguimiento, por su significado moral y por la importancia que tiene su cumplimiento para el agente o una comunidad moral (entre otros criterios).

En la evaluación del principio moral, el ejercicio consiste en revisar si la acción que se deriva del principio es justificable de realizar en circunstancias similares a las descritas en el caso; es decir, si el estudiante señala que toda persona que se encuentra en una situación $x$ tiene la justificación para 
actuar, entonces, todos los casos similares o iguales son justificables (Singer, 1961). El uso de contraejemplos o situaciones hipotéticas se ajusta a este criterio de evaluación, ya que permite apreciar que hay principios éticos que son inadecuados para defender una serie de acciones en la vida social o que simplemente son inaceptables, pues su aceptación haría imposible las relaciones con los otros.

Otra consideración importante en la fase de justificación de las alternativas de acción ante el examen de un dilema ético es revisar el ajuste entre juicios concretos y juicios generales. Esto implica describir adecuadamente los distintos puntos que se pueden defender ante el dilema; examinar la manera cómo, desde distintas creencias morales y desde los principios, podría abordarse la situación moralmente problemática. Esto exige en los estudiantes un ejercicio de ponderación en el cual se asuma en lo posible un punto de vista universal, esto es, una postura que tenga en cuenta no solo mi manera de abordar el problema, sino los posibles puntos de vista de los otros agentes involucrados en el caso. Esta condición requiere que el docente establezca un clima de tolerancia y respeto para la discusión del dilema por parte de los estudiantes, en el cual las opiniones no sean censuradas o descartadas sin un análisis y un debate argumentativo riguroso. La idea es invitar a que el grupo, incluyendo al docente, aprendan juntos del debate que suscita el caso y que la clase no se convierta en una actividad donde autoritariamente se impongan puntos de vista.

\section{Criterios de evaluación para análisis de caso}

Los criterios descritos para analizar los casos en la formación moral pueden ser usados por el docente para orientar y evaluar el ejercicio de aprendizaje ético por parte de sus estudiantes. Los criterios se pueden unificar en una rúbrica como la que a continuación se presenta mediante un ejemplo (Tabla 1). La rúbrica es un instrumento idóneo para evaluar las competencias de deliberación moral que se espera que los estudiantes desarrollen por medio de análisis de dilemas morales. Se puede justificar la idoneidad del instrumento en la medida que permite diseccionar e identificar las tareas que conforman el ejercicio de las competencias de deliberación moral. Los criterios de evaluación están organizados por distintos grados de consecución (insuficiente, regular, bueno, excelente). Cada nivel describe un escenario posible en el cual el docente puede identificar y calificar el desempeño de los estudiantes a la hora de abordar el análisis de casos (Alsina, 2013). La rúbrica puede ofrecer tres ventajas al docente que decida aplicarla.

En primer lugar, la rúbrica sirve para orientar la labor evaluativa para que el docente tenga claridad acerca de qué criterios son relevantes de evaluar con los estudiantes a la hora de implementar los casos como método de enseñanza (Barba \& Romo, 2005).

En segundo lugar, la rúbrica es una alternativa de evaluación ante las deficiencias de los instrumentos tradicionales para evaluar, como lo son los exámenes de opción múltiple que no permiten identificar aspectos de aprendizaje tales como el desarrollo de la capacidad de juicio moral, las habilidades de argumentación y el análisis de circunstancias dilemáticas (Uhl, 1997; Wasserman, 2006).

En tercer lugar, la rúbrica es una herramienta que aumenta la confiabilidad y la validez de la evaluación que hace el docente ante los estudiantes, porque este, al hacer explícitos los criterios a evaluar en su clase, ayuda a que los estudiantes entiendan cuál es el propósito de la enseñanza de la ética a través de casos, tengan consciencia de hasta dónde llegan sus aprendizajes y cuál es el máximo nivel deseable (Alsina, 2013). 
Tabla 1. Matriz de evaluación de análisis de caso

\begin{tabular}{|c|c|c|c|c|}
\hline $\begin{array}{l}\text { Criterio de } \\
\text { evaluación }\end{array}$ & Excelente & Bueno & Regular & Insuficiente \\
\hline $\begin{array}{l}\text { 1. Identificación de } \\
\text { los dilemas morales } \\
\text { que están involucra- } \\
\text { dos en la situación } \\
\text { que presenta el caso. }\end{array}$ & $\begin{array}{l}\text { El estudiante identifica } \\
\text { todos los agentes mo- } \\
\text { rales y sus intereses. } \\
\text { Describe los intereses } \\
\text { en conflicto que están } \\
\text { presentes en el dilema } \\
\text { moral. }\end{array}$ & $\begin{array}{l}\text { El estudiante identifica } \\
\text { a todos los agentes in- } \\
\text { volucrados en el caso. } \\
\text { Describe los intereses } \\
\text { en conflicto pero no lo } \\
\text { hace suficientemente } \\
\text { para lograr su completa } \\
\text { identificación. }\end{array}$ & $\begin{array}{l}\text { El estudiante identifica } \\
\text { parcialmente a los agen- } \\
\text { tes involucrados en el } \\
\text { caso. Describe e identi- } \\
\text { fica parcialmente los in- } \\
\text { tereses morales presen- } \\
\text { tes en el dilema moral. }\end{array}$ & $\begin{array}{l}\text { El estudiante no iden- } \\
\text { tifica a ninguno de los } \\
\text { agentes involucrados } \\
\text { en el caso. Tampoco } \\
\text { describe los intere- } \\
\text { ses morales que están } \\
\text { presentes en el dilema } \\
\text { moral. }\end{array}$ \\
\hline \multirow[b]{2}{*}{$\begin{array}{l}\text { 2. Análisis de las cir- } \\
\text { cunstancias y hechos } \\
\text { relacionados con el } \\
\text { caso. }\end{array}$} & $\begin{array}{l}\text { El estudiante identi- } \\
\text { fica las circunstancias y } \\
\text { hechos más importantes } \\
\text { para el análisis del caso. }\end{array}$ & $\begin{array}{l}\text { El estudiante identifica } \\
\text { algunas circunstancias } \\
\text { y hechos que pueden } \\
\text { ser relevantes para el } \\
\text { análisis del caso pero } \\
\text { se omiten otras circun- } \\
\text { stancias que son impor- } \\
\text { tantes. }\end{array}$ & $\begin{array}{l}\text { El estudiante identifica } \\
\text { unas pocas circunstan- } \\
\text { cias y hechos relaciona- } \\
\text { dos con el caso, pero se } \\
\text { omiten otras circunstan- } \\
\text { cias que son muy rele- } \\
\text { vantes para el desarrollo } \\
\text { del problema. }\end{array}$ & $\begin{array}{l}\text { El estudiante no logra } \\
\text { una identificación de } \\
\text { las circunstancias y } \\
\text { hechos relevantes re- } \\
\text { lacionados con el caso. }\end{array}$ \\
\hline & $\begin{array}{l}\text { Hace un uso apropiado } \\
\text { de la identificación de } \\
\text { las circunstancias y los } \\
\text { hechos a partir de fuen- } \\
\text { tes confiables que en- } \\
\text { riquecen sus argumentos } \\
\text { ante el problema moral } \\
\text { formulado. En el análi- } \\
\text { sis del caso tiene en } \\
\text { cuenta los aspectos fác- } \\
\text { ticos, legales, sociales o } \\
\text { económicos relevantes } \\
\text { para defender una po- } \\
\text { sible alternativa de de- } \\
\text { cisión. }\end{array}$ & $\begin{array}{l}\text { En el análisis del caso se } \\
\text { tienen en cuenta la may- } \\
\text { oría de los aspectos fác- } \\
\text { ticos, legales, sociales o } \\
\text { económicos que pueden } \\
\text { ser relevantes para de- } \\
\text { fender una posible alter- } \\
\text { nativa de decisión. }\end{array}$ & $\begin{array}{l}\text { En el análisis del caso } \\
\text { se usan pocas fuentes } \\
\text { confiables y se tienen } \\
\text { en cuenta solo la mitad } \\
\text { de los aspectos fácti- } \\
\text { cos, legales, sociales o } \\
\text { económicos relevantes } \\
\text { para defender una po- } \\
\text { sible alternativa de de- } \\
\text { cisión. }\end{array}$ & $\begin{array}{l}\text { No se usan fuentes } \\
\text { para sustentar la ar- } \\
\text { gumentación relacio- } \\
\text { nada con el caso. En } \\
\text { el análisis del caso se } \\
\text { omiten aspectos fác- } \\
\text { ticos, legales, socia- } \\
\text { les o económicos que } \\
\text { son relevantes para } \\
\text { defender una posible } \\
\text { alternativa de decisión. }\end{array}$ \\
\hline $\begin{array}{l}\text { 3. Consistencia argu- } \\
\text { mentativa. }\end{array}$ & $\begin{array}{l}\text { La argumentación pro- } \\
\text { puesta por el estudiante } \\
\text { para defender una alter- } \\
\text { nativa de decisión ante } \\
\text { el dilema del caso es } \\
\text { consistente a nivel de } \\
\text { sus premisas y concep- } \\
\text { tos sin cometer contra- } \\
\text { dicciones. }\end{array}$ & $\begin{array}{l}\text { La argumentación pro- } \\
\text { puesta por el estudiante } \\
\text { para defender una alter- } \\
\text { nativa de decisión ante } \\
\text { el dilema del caso en } \\
\text { general puede consid- } \\
\text { erarse en general como } \\
\text { consistente, pero pudo } \\
\text { ser mejor en la relación } \\
\text { entre las premisas o a } \\
\text { nivel conceptual. }\end{array}$ & $\begin{array}{l}\text { La argumentación pro- } \\
\text { puesta por el estudiante } \\
\text { para defender una al- } \\
\text { ternativa de decisión } \\
\text { ante el dilema del caso } \\
\text { en general no logra la } \\
\text { consistencia, porque } \\
\text { presenta errores a nivel } \\
\text { de sus premisas, con- } \\
\text { ceptual o comete alguna } \\
\text { contradicción. }\end{array}$ & $\begin{array}{l}\text { La argumentación pro- } \\
\text { puesta por el estudi- } \\
\text { ante para defender una } \\
\text { alternativa de decisión } \\
\text { ante el dilema del } \\
\text { caso no es consistente } \\
\text { porque comete eviden- } \\
\text { tes o reiterados errores } \\
\text { a nivel de sus premi- } \\
\text { sas, conceptos o cae en } \\
\text { contradicciones. } \\
\end{array}$ \\
\hline $\begin{array}{l}\text { 4. Justificación mor- } \\
\text { al de las alternativas } \\
\text { de decisión. }\end{array}$ & $\begin{array}{l}\text { El estudiante realiza un } \\
\text { detallado examen y eval- } \\
\text { uación de los principios } \\
\text { morales relacionados } \\
\text { con el dilema que de- } \\
\text { scribe el caso. Desarrol- } \\
\text { la un riguroso ejercicio } \\
\text { de ponderación donde } \\
\text { identifica los diferentes } \\
\text { puntos relacionados con } \\
\text { el dilema moral que pre- } \\
\text { senta el caso. }\end{array}$ & $\begin{array}{l}\text { El estudiante realiza un } \\
\text { examen y evaluación } \\
\text { de los principios mo- } \\
\text { rales relacionados con } \\
\text { el dilema que describe } \\
\text { el caso; pero la relación } \\
\text { que establece entre los } \\
\text { principios y el dilema no } \\
\text { está lo suficientemente } \\
\text { argumentada. } \\
\text { Desarrolla un ejercicio } \\
\text { de ponderación donde } \\
\text { identifica algunos pun- } \\
\text { tos relacionados con el } \\
\text { dilema moral que pre- } \\
\text { senta el caso. }\end{array}$ & $\begin{array}{l}\text { El estudiante realiza un } \\
\text { examen y evaluación } \\
\text { de los principios mo- } \\
\text { rales relacionados con } \\
\text { el dilema que describe } \\
\text { el caso; pero la relación } \\
\text { que establece entre los } \\
\text { principios y el dilema es } \\
\text { confusa o errónea. } \\
\text { Desarrolla un ejercicio } \\
\text { de ponderación donde } \\
\text { identifica algunos pun- } \\
\text { tos de vista (omitiendo } \\
\text { lo más importantes) re- } \\
\text { lacionados con el dilema } \\
\text { moral que presenta el } \\
\text { caso. }\end{array}$ & $\begin{array}{l}\text { El estudiante no reali- } \\
\text { za un examen y evalu- } \\
\text { ación de los principios } \\
\text { morales relacionados } \\
\text { con el dilema que de- } \\
\text { scribe el caso. No de- } \\
\text { sarrolla un ejercicio } \\
\text { de ponderación donde } \\
\text { identifique los puntos } \\
\text { de vista relacionados } \\
\text { con el dilema moral } \\
\text { que presenta el caso. }\end{array}$ \\
\hline
\end{tabular}




\section{Conclusiones}

Como se mencionó en la introducción del artículo, el proceso la formación moral se puede mejorar con la discusión de casos que presenten situaciones reales complejas. Esta metodología, que ha sido usada extensivamente en otras disciplinas como el derecho, la administración y la medicina, pone en juego una serie de competencias de deliberación como la capacidad de identificar los conflictos y los actores envueltos en la situación, la capacidad de establecer los posibles cursos de acción y la capacidad de determinar las limitantes naturales, sociales, históricas, económicas y culturales.

El propósito central del artículo era presentar los criterios que han sido seleccionados y elaborados a partir de consultas con docentes que orientan la asignatura de ética y valores tanto en la educación básica, media y superior. Esos criterios son esenciales para guiar el análisis y la evaluación de casos dilemáticos que ayuden a los estudiantes al desarrollo de su carácter moral; entendiendo ese carácter como la capacidad que tiene el individuo de evaluar y prever consecuencias de sus acciones. En ese sentido, la formación moral debe apuntar a que los estudiantes se familiaricen con seleccionar y elegir los cursos de acción, seguir reglas lógicas en la toma de decisiones y, por ende, argumentar de manera coherente sus creencias, acciones y compromisos (Cabezas, 2014).

Otro valor importante del uso de casos en la discusión moral es que promueve en los estudiantes su capacidad de justificación a partir de principios que involucran acciones y emociones que sirven para identificar y defender cuándo una acción es o no moralmente buena. El uso de casos ayuda al docente a identificar si en el aula esa capacidad de justificación se está fortaleciendo y evaluar en qué nivel de desarrollo del juicio moral se encuentran sus estudiantes.

Cabe aclarar que los aspectos propuestos en el artículo suponen que los estudiantes cuentan con habilidades lógicas, de interpretación y de comprensión que se esperaría encontrar en una población estudiantil de últimos grados de educación básica y media o en primeros semestres de educación superior. Ante esos supuestos, es pertinente señalar que una adecuada apropiación de los dilemas para el desarrollo moral debe tener en cuenta las etapas y niveles propuestos por Kohlberg, de tal manera que el docente proponga casos que sean adecuadas tanto a la edad como al nivel de desarrollo moral que presentan sus estudiantes.

En principio, se espera que los criterios propuestos sean universales en el sentido que se puedan exigir y aplicar a cualquier población estudiantil de últimos grados de educación básica y media o de educación superior, sin que, en principio, sea relevante discriminar variables socioculturales que puedan favorecer o afectar la manera como los estudiantes abordan los casos. Sin embargo, no cabe descartar el que factores emocionales vinculados al cultivo de sentimientos morales como la simpatía, la culpa o la vergüenza influyan en la manera en que los estudiantes se identifican con los personajes del caso o sean capaces de estar más inclinados por ciertas alternativas de acción.

\section{Lista de referencias}

Alsina, J. (2013). Introducción. En P. Alsina (Coord.), Rúbricas para evaluación de competencias. Barcelona: Octaedro.

Anaya, R., \& Ocampo, E. (2016). Formación de ciudadanía en la escuela dentro de la transición democrática: ¿promover escalas de valores o el desarrollo del razonamiento moral? Reice. Revista Iberoamericana sobre Calidad, Eficacia y Cambio en Educación, 14(1), 5-35. doi:10.15366/ reice2016.14.1.001

Barba, B., \& Romo, J. M. (2005). Desarrollo del juicio moral en la educación superior. Revista Mexicana de Investigación Educativa, 10(24), 67-92.

Bermúdez, A., \& Jaramillo, R. (2000). El análisis de dilemas morales una estrategia pedagógica para el desarrollo de la autonomía moral. Bogotá, D. C.: Secretaría de Educación Distrital.

Beuchamp, T., \& Childress, J. (2002). Principios de ética biomédica. Barcelona: Masson. 
Cabezas, M. (2014). Ética y emoción: el papel de las emociones en la justificación de nuestros juicios morales. Madrid: Plaza y Valdés.

Carrillo, I. (1982). Discusión de dilemas morales y desarrollo progresivo del juicio moral. CL\&E: Comunicación, Lenguaje y Educación, 15, 55-62.

Copi, I., \& Cohen., C. (1990). Introducción a la lógica. México, D. F.: Limusa.

Erramouspe, P. (2004). Un cambio de mirada en la didáctica de la argumentación moral. En G. Schujman (Coord.), Formación ética y ciudadana: un cambio de mirada (45-78). Barcelona: Octaedro.

Estrada, A., \& Alfaro, K. (2015). El método de casos como alternativa pedagógica para la enseñanza de la bibliotecología y las ciencias de la información. Bibliotecológica, 29(65), 195-212. doi:10.15517/eci.v5i2.19736

Ewing, D. W. (1990). Inside the Harvard Bussiness School. Nueva York: Random House.

Habermehl, L. (1976). The susceptibility of moral claims to reasoned assessment. En L. Habermehl (Ed.), Morality in the modern world (pp. 68-77). Belmont: Dickenson Publishing.

Hernández, M., González, V., \& Pérez, M. (2015). El razonamiento moral como elemento constitutivo de la competencia ética: dilemas reales e hipotéticos en profesores de educación secundaria en México. Revista Complutense de Educación, 26(1), 183-201. doi:10.5209/rev_RCED.2015.v26. $\mathrm{n} 1.42900$

Hirsch, A. (2006). Construcción de un estado de conocimiento de valores profesionales en México. Revista Electrónica de Investigación Educativa, 8(2), 5-23. Recuperado de: http://redie. uabcmx/vol8no2/contenido-hirsch2.html

Kohlberg, L. (1984). Essays on moral development, II. Nueva York: Harper \& Row.

Lemmon, J. (1962). Moral dilemmas. Philosophical Review, 71, 139-158. doi:10.2307/2182983

Linde, A. (2009). ¿Tienen vigencia los dilemas clásicos de Kohlberg en educación moral?: un estudio de campo. Campo Abierto, 28, 137-149.

Martínez, L., Navarro, Y., \& Pérez, L. (2015). Razonamiento moral y ético en estudiantes de educación secundaria a partir de cuestiones sociocientíficas. Uni-Pluri/Versidad, 15, pp. 76-86.

Meza, J. L. (2008). Los dilemas morales: una estrategia didáctica para la formación del sujeto moral en el ámbito universitario. Revista Actualidades Pedagógicas, 52, 13-24. doi:10.19052/ap.1324

Molina, A. (2010). Un argumento en contra de la coherencia. Revista Diálogos de Saberes, 11, 193217.

Noam, G. G. (1985). Stage, phase, and style: The developmental dynamics of the self. En M. W. Berkowitz, \& Oser, F. (Eds.), Moral education: Theory and application (pp. 321-346). Hillsdale: L. Erlbaum and Associates.

Ortega, P., \& Mínguez, P. (1992). La educación moral en la infancia y adolescencia. Teoría de la Educación, 4, 151-163.

Puig, J. \& Martín, X. (2007). Competencias en autonomía e iniciativa personal. Madrid: Alianza.

Puig, J., \& Martín, X. (2015). Para un currículum de educación en valores. Folios, 41, 7-22. doi:10.17227/01234870

Rest, J. (1979). Development in judging moral issues. Minneapolis: University of Minnesota.

Singer, G. (1961). Generalization in ethics. Nueva York: Alfred A. Knopf.

Toulmin, S., Rieke, R., \& Janik, A. (1979). An introduction to reasoning. Nueva York: Macmillan.

Uhl, S. (1997). Los medios de educación moral y su eficacia. Barcelona: Herder.

Velásquez, M. (2000). Ética en los negocios: conceptos y casos. México, D. F.: Pearson.

Wassermann, S. (2006). El estudio de casos como método de enseñanza. Buenos Aires: Amorrortu. 